\title{
Shell rupture threshold, fragmentation threshold, Blake threshold
}

\author{
Michiel Postema*, Nico de Jong ${ }^{\dagger}$, Georg Schmitz* \\ *Institute for Medical Engineering, Ruhr-Universität Bochum, Bochum, Germany \\ ${ }^{\dagger}$ Physics of Fluids group, Faculty of Science and Technology, University of Twente, Enschede, The Netherlands
}

\begin{abstract}
The disruption of contrast agent microbubbles has been implicated in novel techniques for high-MI imaging and local drug delivery. At MI $>0.6$, microbubble fragmentation has been observed with thin-shelled agent $(\approx 10 \mathrm{~nm})$, and shell rupture with thick-shelled agent $(\approx 250 \mathrm{~nm})$. To predict the disruption of these nanoshelled microbubbles, destruction thresholds have been under investigation. In several studies, the Blake threshold pressure was associated with microbubble destruction. The Blake threshold pressure is the peak rarefactional acoustic pressure at which the critical Blake radius is reached, approximately twice the equilibrium radius, above which a bubble behaves like an inertial cavity. We studied the acoustic pressures at which a thin-shelled microbubble fragments and those at which a thick-shelled microbubble cracks. More specifically, we investigated the validity of the Blake threshold for these phenomena. The oscillating and fragmenting behavior of microbubbles with a $10 \mathrm{~nm}$ shell was simulated at a driving frequency of $0.5-2 \mathrm{MHz}$, using a modified Rayleigh-Plesset equation and assuming that fragmentation occurs when the kinetic energy of the microbubble surpasses the instantaneous bubble surface energy. For microbubbles with radii between 1 and $6 \mu \mathrm{m}$, the fragmentation thresholds lie between 20 and $200 \mathrm{kPa}$. Generally, the critical radius is much smaller than twice the equilibrium radius. The moment of break-up during the collapse phase is in agreement with highspeed optical observations that were presented previously.

Furthermore, the shell rupture behavior of microbubbles with a thick shell was analyzed for quasistatic pressure changes (relatively low ultrasonic frequencies), assuming that the shell obeys Hooke's Law. The rupture threshold pressure of $-80 \mathrm{kPa}$ had been determined from acoustical data. For shells with the typical Young's modulus $2 \mathrm{MPa}$ and Poisson ratio 0.5, this is in agreement with the observation that the maximal excursion upon rupture of such bubbles is smaller that $0.3 \mu \mathrm{m}$.

In conclusion, we may state that the Blake threshold is neither a good estimator for the fragmentation, nor for the rupture of contrast agent microbubbles.
\end{abstract}

\section{INTRODUCTION}

Ultrasound contrast agents consist of encapsulated gas bubbles with equilibrium radii $R_{0}$ up to $5 \mu \mathrm{m}$. These microbubbles are small enough to be transported intravascularly and to pass through capillary vessels. Because their resonance frequencies coincide with those applied in ultrasonic imaging, they are suitable markers for the detection of perfused areas [1]. Predicting the dynamic behavior of ultrasound insonified encapsulated microbubbles has been of much clinical interest. For perfusion measurements, a technique named flash-echo has been proposed. A burst of high-MI ultrasound is to disrupt the contrast agent microbubbles, supposedly resulting in a strong scattering signal that is visible on the B-mode image: the flash. The absence of this strong response in parts of the B-mode image indicates a (too) low perfusion [2]. For therapeutic purposes, it has been proposed to use encapsulated microbubbles that act as a vehicle to carry the drug to a region of interest. By applying a burst of high-MI ultrasound, the shell encapsulating the gas can be cracked. Consequently, the drug is released at the region of interest, only. This process is generally referred to as (ultra)sonic cracking.

To enhance flash-echo techniques and to develop therapeutic release methods, the ultrasonic disruption of contrast agent microbubbles has to be understood. Previously, we presented a quantitative overview of contrast agent microbubble disruption mechanisms, based on high-speed optical observations [3]. We found that fragmentation is the most occurring disruption mechanism for microbubbles with a thin shell $(\approx 10 \mathrm{~nm})$. Fragmentation is the fission of a microbubble into a number of smaller microbubbles. Ultrasonic cracking has been primarily observed with thick-shelled microbubbles $(\approx 250 \mathrm{~nm})$.

To predict the disruption of these nanoshelled microbubbles, destruction thresholds have been under investigation. In several studies, the Blake threshold pressure was associated with microbubble destruction. We studied the acoustic pressures at which a thin-shelled microbubble fragments and those at which a thick-shelled microbubble cracks. More specifically, we investigated the validity of the Blake threshold for these phenomena. Furthermore, the shell rupture behavior of microbubbles with a thick shell was analyzed for quasistatic pressure changes.

\section{THEORY}

\section{A. Cavitation}

In this section, we describe the growth of a microbubble in an infinite liquid subjected to an ultrasonic field. If the insonifying frequency is much lower than the resonance frequency of the microbubbles, the pressure in the liquid $p_{\mathrm{L}}$ changes very slowly and uniformly compared to the natural time scale of the microbubble [4], [5]. The radius of a bubble $R$ in response to quasistatic changes in the liquid pressure is described by [5]:

$$
p_{\mathrm{L}}=\left(p_{0}^{\infty}-p_{\mathrm{v}}+\frac{2 \mu}{R_{0}}\right)\left(\frac{R_{0}}{R}\right)^{3 \gamma}+p_{\mathrm{v}}-\frac{2 \mu}{R} .
$$

Here, $p_{0}^{\infty}$ is the static pressure of the liquid, $p_{\mathrm{v}}$ is the vapor pressure, $R_{0}$ is the equilibrium radius of the microbubble, $\gamma$ is the polytropic exponent, and $\mu$ is the surface tension. 
Figure 1 shows the right-hand-side of eq. (1), for different $R_{0}$. Each curve has a minimum in $\left(R_{\mathrm{c}}, p_{\mathrm{L}_{\mathrm{c}}}\right)$, where $R_{\mathrm{c}}$ is the
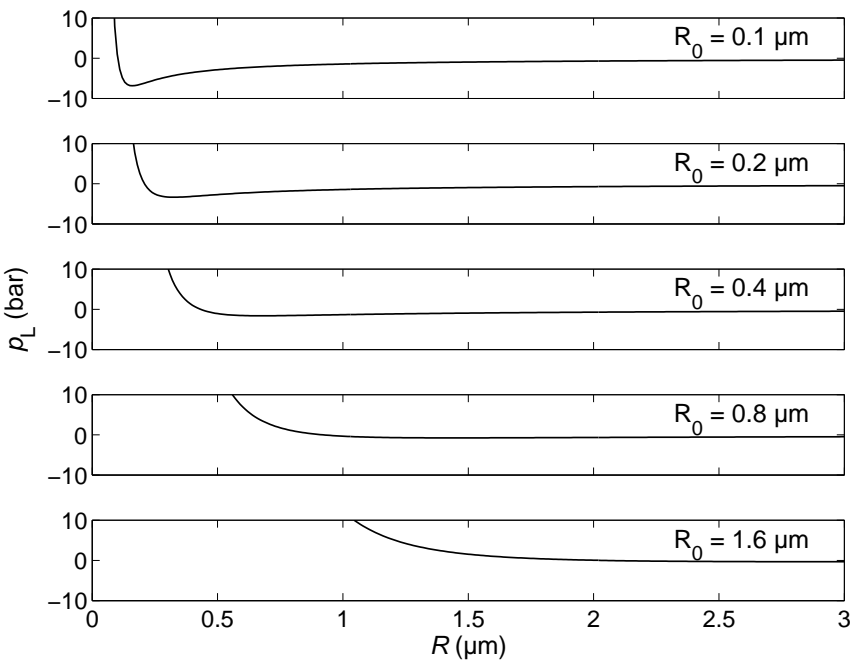

Fig. 1. Solutions of eq. (1) for different equilibrium radii $0.1 \leq R_{0} \leq$ $1.6 \mu \mathrm{m}$, taking $p_{0}^{\infty}=1 \mathrm{~atm}, \gamma=1.4$, and $\mu=0.072 \mathrm{~kg} \mathrm{~s}^{-2}$.

critical Blake radius and $p_{\mathrm{L}_{\mathrm{c}}}$ is the critical liquid pressure. The region to the right of the Blake radius represents unstable equilibrium conditions [4], [6]. If the liquid pressure is lowered until it reaches a value below $p_{\mathrm{L}}$, no equilibrium radius exists [5], resulting in explosive growth of the bubble, much larger than $R_{0}$. The ambient pressure eventually increases again, during the ultrasonic compression phase, causing the bubble to collapse violently [7]. The Blake radius has been approximated by

$$
R_{\mathrm{c}} \approx 2 R_{0} .
$$

At relatively high acoustic amplitudes (mechanical index $\mathrm{MI} \approx 0.6$ ), thin-shelled microbubbles may expand to more than 10-fold their initial surface areas before coalescing [8]. By the time of maximal expansion, therefore, the shell has ruptured, leaving newly formed clean free interfaces [8]. As such, the elastic properties of the shell will have diminished, resulting in maximal expansions similar to free gas bubbles [9]. Therefore, we neglect the influence of the shell in this section.

During the initial part of the collapse the acceleration, $\ddot{R}$ is negative. This sign changes as the gas inside the bubble begins to be compressed, and the rebound begins [7]. Microbubble fragmentation has been expected and observed close to this moment, when $\ddot{R}=0$ [3], [10].

\section{B. Fragmentation}

The oscillating behavior of an encapsulated microbubble has been described by modified RPNNP equation [11]:

$$
\begin{aligned}
& \rho R \ddot{R}+\frac{3}{2} \rho \dot{R}^{2}=p_{\mathrm{g} 0}\left(\frac{R_{0}}{R}\right)^{3 \gamma}+p_{\mathrm{v}}-p_{0}^{\infty} \\
& -\frac{2 \mu}{R}-2 S_{\mathrm{p}}\left(\frac{1}{R_{0}}-\frac{1}{R}\right)-\delta \omega \rho R \dot{R}-p_{\mathrm{a}}(t),
\end{aligned}
$$

where $p_{\mathrm{g} 0}$ is the initial gas pressure inside the bubble $p_{\mathrm{g} 0}=p_{0}^{\infty}-p_{\mathrm{v}}+\frac{2 \mu}{R_{0}}, p_{\mathrm{a}}(t)$ is the acoustic pressure in time, $S_{\mathrm{p}}$ is the shell stiffness parameter, $\delta$ is the total damping coefficient, $\rho$ is the liquid density, and $\omega$ is the angular driving frequency. The total damping coefficient is a summation of the damping coefficient due to friction of the shell, the damping coefficient due to radiation, the damping coefficient due to heat conduction, and the damping coefficient due to the viscosity of the surrounding liquid [11].

The number of fragments $N$ into which a microbubble breaks up, is related to the dominant spherical harmonic oscillation mode $n$ by [7], [9]:

$$
N \approx n^{3} \text {. }
$$

Mode 2 oscillations have been observed with lipidencapsulated microbubbles, leading to fragmentation into 8 newly formed microbubbles [9].

The kinetic energy of a single microbubble in an infinite fluid is given by [7]:

$$
E_{\mathrm{k}}=2 \pi \rho R^{3} \dot{R}^{2} .
$$

The resulting microbubble fragments contain more surface free energy $\sum_{i} E_{\mathrm{f}, i}$ than the single bubble prior to fragmentation $E_{\mathrm{s}}$ :

$$
\sum_{i=1}^{N} E_{\mathrm{f}, i} \approx \frac{4}{3} \pi R_{\mathrm{f}}^{2} \mu N \approx \frac{4}{3} \pi R^{2} \mu N^{\frac{1}{3}}=N^{\frac{1}{3}} E_{\mathrm{s}} .
$$

We assumed that fragmentation will only occur if and only if [12]:

$$
E_{\mathrm{k}}>\left(\sum_{i=1}^{N} E_{\mathrm{f}, i}-E_{\mathrm{s}}\right) .
$$

\section{Ultrasonic cracking}

The shell stiffness parameter is given by [13]:

$$
S_{\mathrm{p}}=\frac{8 \pi E h_{\mathrm{s}}}{1-\nu}
$$

where $E$ is Young's modulus, $h_{\mathrm{s}}$ is the shell thickness, and $\nu$ is the Poisson ratio. For albumin and lipid nanoshells, we take $0.499<\nu<0.500 . S_{\mathrm{p}}$ can be estimated from optical observations of radius-time curves or from acoustical data using the relation [14]:

$$
\omega_{\mathrm{s}}^{2}=\omega_{\mathrm{r}}^{2}+\frac{S_{\mathrm{p}}}{4 \pi R_{0}^{3} \rho},
$$

where $\omega_{\mathrm{s}}$ is the angular resonance frequency of the nanoshelled microbubble, $\omega_{\mathrm{r}}$ is the angular resonance frequency of an unencapsulated microbubble of the same size.

$$
\omega_{\mathrm{r}}\left[\mathrm{rad} \mathrm{s}^{-1}\right] \approx \frac{6.5 \pi\left[\mathrm{rad} \mathrm{m} \mathrm{s}^{-1}\right]}{R_{0}[\mathrm{~m}]} .
$$

The critical stress at which a shell ruptures $\sigma_{\mathrm{c}}$, is related to Young's modulus by [9]:

$$
\sigma_{\mathrm{c}} \approx E \epsilon_{\mathrm{c}},
$$

where $\epsilon_{\mathrm{c}}$ is the critical lateral shell deformation. For most biomaterials, $\epsilon_{\mathrm{c}}<0.5$. 


\section{METHODS}

We simulated the oscillating behavior of free microbubbles with various sizes in a harmonic acoustic field:

$$
p_{\mathrm{a}}(t)=p_{\mathrm{a}}^{-} \sin \omega t .
$$

Equations (3)-(6) were computed with MATLAB ${ }^{\circledR}$ (The MathWorks, Inc., Natick, MA) programs. The following fixed parameters were used: $c=1480 \mathrm{~m} \mathrm{~s}^{-1}, p_{0}^{\infty}=1$ atm, $p_{\mathrm{v}}=2.33 \mathrm{~Pa}, \gamma=1.4, \eta=0.001 \mathrm{~Pa} \mathrm{~s}, \rho=998 \mathrm{~kg} \mathrm{~m}^{3}$, and $\mu=0.072$ $\mathrm{kg} \mathrm{s}^{-2}$. The acoustic amplitudes modeled correspond to $\mathrm{MI} \ll 2$ (well within the clinical diagnostic range). Here, we focus on simulated driving frequencies of $0.5 \mathrm{MHz}$ and 2.0 $\mathrm{MHz}$, because our optical interrogation was undertaken using these ultrasonic frequencies.

For microbubble radii $0.2<R_{0}<12.0 \mu \mathrm{m}$, the critical acoustic pressures $p_{\mathrm{c}}$ were computed, above which eq. (7) holds. For comparison with the Blake critical radius, the maximal microbubble radii $R_{\max }\left(R_{0}, p_{\mathrm{c}}\right)$ were computed using the RPNNP-equation, and divided by the initial radii $R_{0}$.

Furthermore, we computed the shell properties of three ultrasound contrast agents from their acoustic resonance frequencies, using equations (8)-(11).

\section{RESUlTS AND Discussion}

An example showing high-speed photographs of microbubble fragmentation is presented in Fig. 2. Fragmentation occurs during collapse. In most observations of fragmenting contrast agent microbubbles, other bubbles can be observed close to the fragmenting bubble. If nearby microbubbles are close enough to an oscillating microbubble, these may induce surface instabilities that have been associated with fragmentation. After fragmentation, microbubble fragments have been observed to coalesce (merge), reducing the surface energy. An image sequence of the coalescence of free (not encapsulated) microbubble fragments has been presented in [15].

An example of simulated oscillating behavior of a microbubble is shown in Fig. 3. The relatively slow expansion is followed by a rapid collapse. The collapse generates a high acoustic pressure [12]. This way, the collapsing microbubbles account for the flash. Close to collapse, the kinetic energy of the microbubble becomes higher than the surface energy, as hypothesized. This is the oscillation phase where microbubble break-up has been observed.

The maximal expansion radii at the critical pressures normalized by the initial radii are demonstrated in the right frame of Fig. 4. In contradiction to the assumption that the Blake critical radius is a good approximation for a fragmentation threshold, our simulations show $R_{\max } / R_{0} \ll 2$ for most microbubbles.

For simplicity, here, we introduce the variable $a$, which gives the bubble excursion $a(t)=R(t)-R_{0}$. For microbubbles with a thick, stiff nanoshell, like Quantison ${ }^{\mathrm{TM}}, a(t) \ll$ $R_{0}$. From high-speed optical observations, we derived that $\max (a(t)) \leq \mathcal{R}$, where $\mathcal{R} \approx 0.3 \mu \mathrm{m}$ is the resolution of the optical system. From the difference in resonance

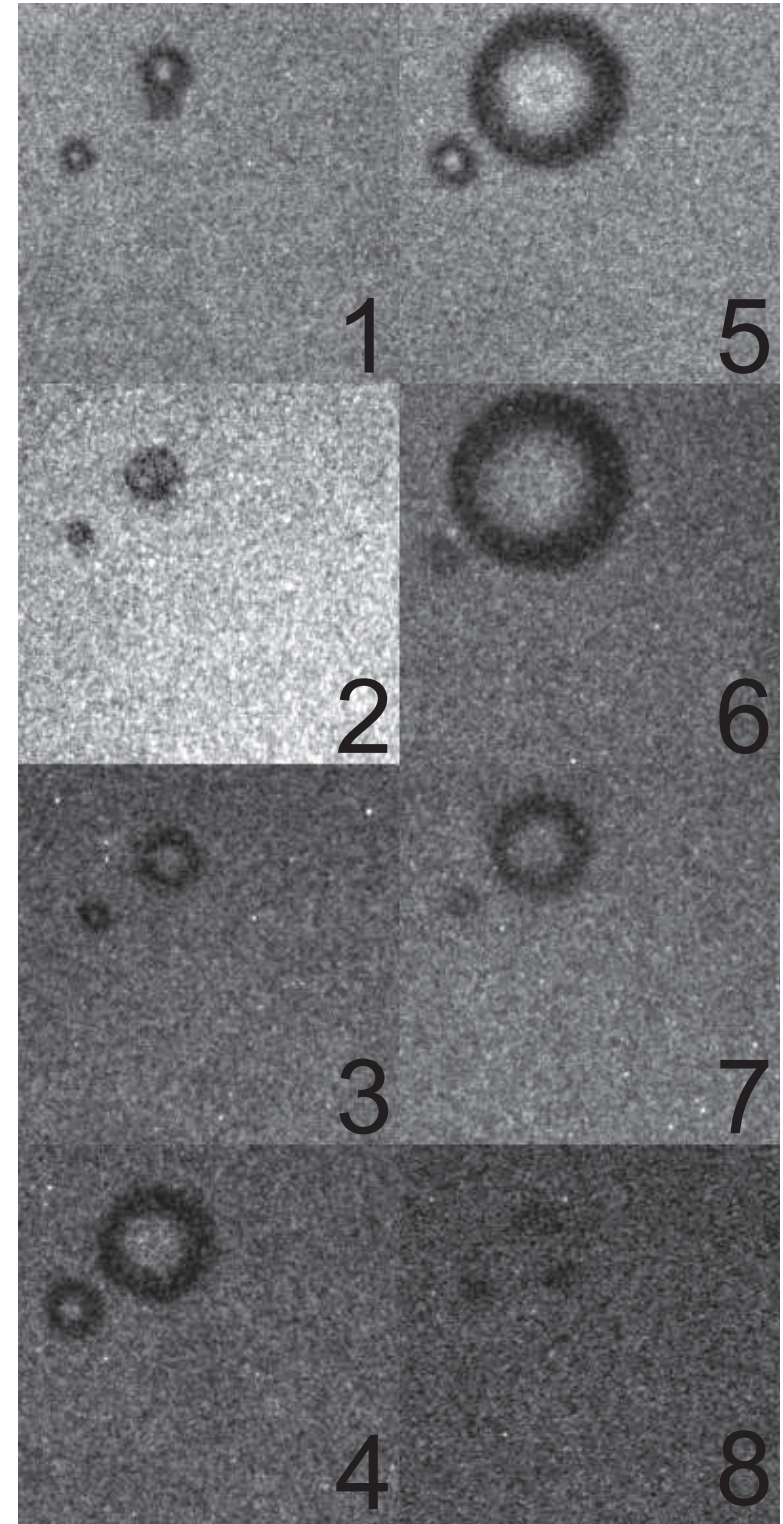

Fig. 2. Two optical image sequences showing fragmentation of lipidencapsulated contrast agent microbubbles (Bracco Research SA, Genève, Switzerland) during insonification at $0.5 \mathrm{MHz}$ and $\mathrm{MI}<1$. The frames were captured at 3 million frames per second. Frames 1 have been taken prior to ultrasound arrival. The other seven frames in a sequence cover one ful ultrasonic cycle. Each frame corresponds to a $30 \times 30 \mu \mathrm{m}^{2}$ area. $R_{0}=1.5 \mu \mathrm{m}$, $R_{\max }=7 \mu \mathrm{m}, N>7$. An $R_{0}=1 \mu \mathrm{m}$ microbubble is present to the lower left at $r=10 \mu \mathrm{m}$ distance. The images were captured at the Department of Experimental Echocardiography, Thoraxcentre, Erasmus MC, Rotterdam, The Netherlands.

frequency between Quantison ${ }^{\mathrm{TM}}$ and free gas microbubbles, we determined $S_{\mathrm{p}}=25 \mathrm{~kg} \mathrm{~s}^{-2}$ and $E=2 \times 10^{6} \mathrm{~Pa}$. The critical stress of Quantison ${ }^{\mathrm{TM}}$ is $\left|\sigma_{\mathrm{c}}\right| \geq 80 \mathrm{kPa}$ [16], and thus $\epsilon_{\mathrm{c}} \geq 0.4$. Taking into account that $\epsilon_{\mathrm{c}}<0.5$ and $\left\langle R_{0}\right\rangle=1.6 \mu \mathrm{m}$, it follows that:

$$
\max (a(t)) \approx 0.3 \mu \mathrm{m}=\mathcal{R} .
$$

The shell properties of three contrast agents has been summarized in Tabel I. SonoVue ${ }^{\mathrm{TM}}$ has a thin monolayer lipid shell, Quantison ${ }^{\mathrm{TM}}$ has a thick albumin shell, and Albunex $\AA$ 
TABLE I

ELASTIC PROPERTIES OF THREE CONTRAST AGENTS

\begin{tabular}{lcccc}
\hline & $\begin{array}{c}\omega_{\mathrm{r}} \\
{\left[2 \pi \times 10^{6} \mathrm{rad} \mathrm{s}^{-1}\right]}\end{array}$ & $\begin{array}{c}\left\langle R_{0}\right\rangle \\
{[\mu \mathrm{m}]}\end{array}$ & $\begin{array}{c}S_{\mathrm{p}} \\
{\left[\mathrm{kg} \mathrm{s}^{-2}\right]}\end{array}$ & $\begin{array}{c}E^{*} \\
{\left[10^{6} \mathrm{~Pa}\right]}\end{array}$ \\
\hline Albunex® & 2 & 4.0 & 10 & 2 \\
Quantison $^{\mathrm{TM}}$ & 4 & 1.6 & 25 & 2 \\
SonoVue $^{\mathrm{TM}}$ & 3 & 1.0 & 1.1 & 2 \\
\hline
\end{tabular}

has a thin albumin shell.

\section{CONCLUSIONS}

Fragmentation occurs exclusively during the collapse phase. We hypothesize that fragmentation will only occur if and only if the kinetic energy of the collapsing microbubble is greater than the instantaneous bubble surface energy. From our simulations it follows that the Blake critical radius is not a good approximation for a fragmentation threshold.

The shell rupture behavior of microbubbles with a $250 \mathrm{~nm}$ thin elastic shell was analyzed for quasistatic pressure changes (relatively low ultrasonic frequencies), assuming that the shell obeys Hooke's Law. The rupture threshold pressure of $-80 \mathrm{kPa}$ had been determined from acoustical data. For shells with the typical Young's modulus $2 \mathrm{MPa}$ and Poisson ratio 0.5 , this is in agreement with the observation that the maximal excursion upon rupture of such bubbles is smaller than $0.3 \mu \mathrm{m}$.

In conclusion, we may state that the Blake threshold is neither a good estimator for the fragmentation, nor for the rupture of contrast agent microbubbles.

\section{REFERENCES}

[1] P. N. Burns, P. Hilpert, and B. B. Goldberg, "Intravenous contrast agent for ultrasound Doppler: In vivo measurement of small tumor vessel
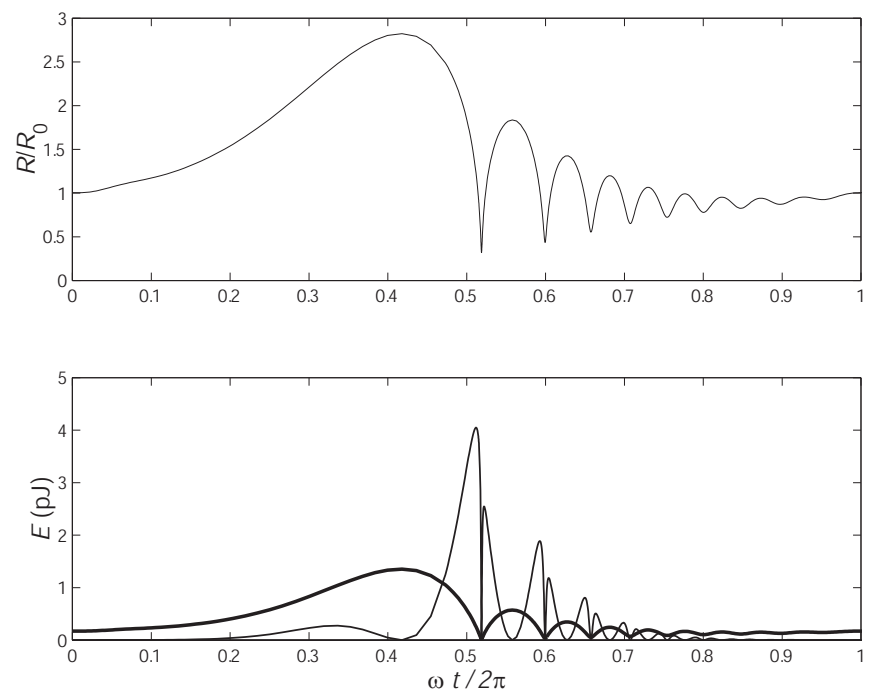

Fig. 3. Simulation of the oscillation behavior of an $R_{0}=0.75 \mu \mathrm{m}$ microbubble, during insonification at $0.5 \mathrm{MHz}$ and $p_{\mathrm{a}}^{-}=210 \mathrm{kPa}$. In the lower frame, the bold line represents the difference in surface energy $\sum_{i}^{N} E_{\mathrm{f}, i}-E_{\mathrm{s}}$ between $N$ fragments and a whole bubble, whereas the thin line represents the kinetic energy $E_{\mathrm{k}}$ of the bubble. Here, $\sum_{i}^{N} E_{\mathrm{f}, i}$ was computed for $N=8$.

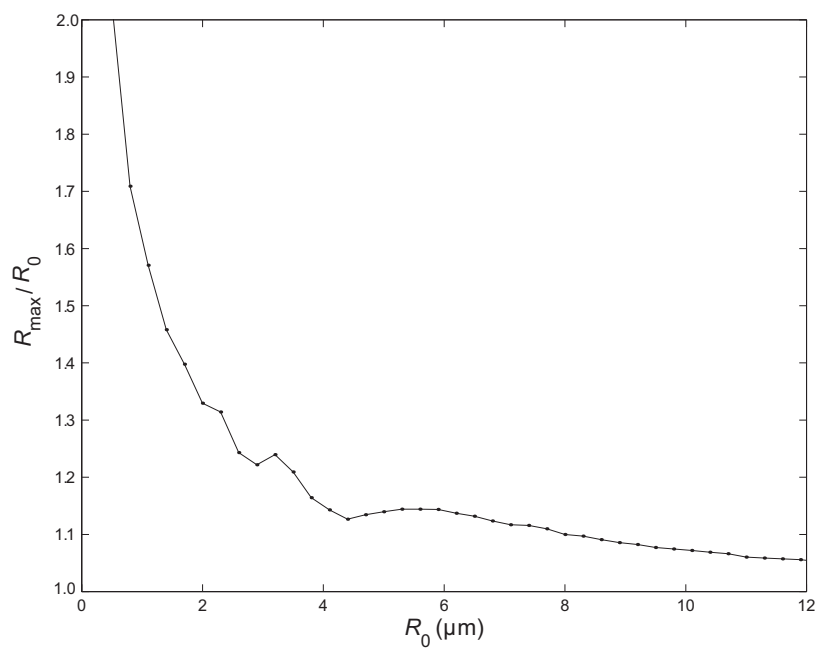

Fig. 4. Relative microbubble excursion as a function of initial radius.

dose-response," Proc. IEEE Eng. Med. Biol. Soc., vol. 12, no. 1, pp. 322-324, 1990.

[2] K. Wei, A. R. Jayaweera, S. Firoozan, A. Linka, D. M. Skyba, and S. Kaul, "Quantification of myocardial blood flow with ultrasoundinduced destruction of microbubbles administered as a constant venous infusion," Circulation, vol. 97, pp. 473-483, 1998.

[3] M. Postema, A. Bouakaz, C. T. Chin, and N. de Jong, "Optical observations of ultrasound contrast agent destruction," Acta Acust. united Acust., vol. 89, p. 728, 2003.

[4] C. E. Brennen, Cavitation and bubble dynamics. New York: Oxford University Press, Inc, 1995.

[5] A. Harkin, A. Nadim, and T. J. Kaper, "On acoustic cavitation of slightly subcritical bubbles," Phys. Fluids, vol. 11, no. 2, pp. 274287, 1999.

[6] D. Or and M. Tuller, "Reply to comment by N. Kartal Toker, John T. Germaine, and Patricia J. Culligan on "Cavitation during desaturation of porous media under tension"," Water Resour. Res., vol. 35, no. 7, pp. 1949-1964, 1999.

[7] C. E. Brennen, "Fission of collapsing cavitation bubbles," Proc. CAV 2001 4th Int. Symp. Cavitation, 2001

[8] M. Postema, P. Marmottant, C. T. Lancée, S. Hilgenfeldt, and N. de Jong, "Ultrasound-induced microbubble coalescence," Ultrasound Med. Biol., vol. 30, no. 10, pp. 1337-1344, 2004.

[9] M. Postema, A. van Wamel, C. T. Lancée, and N. de Jong, "Ultrasound-induced encapsulated microbubble phenomena," Ultrasound Med. Biol., vol. 30, no. 6, pp. 827-840, 2004.

[10] J. E. Chomas, P. Dayton, D. May, and K. Ferrara, "Threshold of fragmentation for ultrasonic contrast," J. Biomed. Opt., vol. 6, no. 2, pp. 141-150, 2001.

[11] N. de Jong, Acoustic properties of ultrasound contrast agents. Rotterdam: N de Jong, 1993.

[12] M. Postema and G. Schmitz, "Ultrasonic fragmentation of microbubbles: a theoretical approach of the flash in flash-echo," Proc. IEEE Eng. Med. Biol. Soc., vol. accepted, 2005.

[13] N. de Jong, L. Hoff, T. Skotland, and N. Bom, "Absorption and scatter of encapsulated gas filled microspheres: theoretical considerations and some measurements," Ultrasonics, vol. 30, no. 2, pp. 95-103, 1992.

[14] N. de Jong, A. Bouakaz, and P. Frinking, "Basic acoustic properties of microbubbles," Echocardiography, vol. 19, no. 3, pp. 229-240, 2002.

[15] M. Postema, P. Marmottant, C. T. Lancée, M. Versluis, S. Hilgenfeldt, and N. de Jong, "Ultrasound-induced coalescence of free gas microbubbles," Proc. IEEE Ultrason. Symp., pp. 1-4, 2004.

[16] P. J. A. Frinking and N. de Jong, "Acoustic modeling of shellencapsulated gas bubbles," Ultrasound Med. Biol., vol. 24, no. 4, pp. 523-533, 1998.

Michiel Postema e-mail: postema@ieee.org 